\title{
Correction to: Reactive synthesis without regret
}

\author{
Paul Hunter $^{1}$ - Guillermo A. Pérez ${ }^{1}$ (D) Jean-François Raskin ${ }^{1}$ \\ Published online: 20 October 2021 \\ (c) Springer-Verlag GmbH Germany, part of Springer Nature 2021
}

\section{Correction to: Acta Informatica (2017) 54:3-39 https://doi.org/10.1007/s00236-016-0268-z}

We point to inaccuracies in the article "Reactive Synthesis Without Regret". We are very thankful to Udi Boker and Karoliina Lehtinen for notifying us about these inaccuracies.

- There is text in pages 9-10 that is duplicated. The second copy of "Consider a [...] (instead of the minimal)." after Lemma 2 should be removed.

- In contrast to what is stated in the definitions, throughout the article we assume games and automata can have rational weights on edges and transitions instead of integer weights. This is of technical importance for some of the proofs-even though the hardness statements still hold for integer-weighted games and automata if $r$ is part of the input.

- Theorem 5 is only true for strictly positive values of $r$ and the proof needs minor changes. Namely, the left-hand-side gadget wherein Eve does not choose to play, should get a value of 2 . Then, the regret will be at most 1 if and only if the formula is satisfiable. This is easy to generalize to other nonzero positive values of $r$.

Publisher's Note Springer Nature remains neutral with regard to jurisdictional claims in published maps and institutional affiliations.

The original article can be found online at https://doi.org/10.1007/s00236-016-0268-z.

Guillermo A. Pérez

guillermoalberto.perez@uantwerpen.be

1 Département d'Informatique, Université Libre de Bruxelles (ULB), Brussels, Belgium 